\title{
Comparisons of the joint moments between leading and trailing limb in young adults when stepping over obstacles
}

\author{
Hao-Ling Chen, Tung-Wu Lu* \\ Institute of Biomedical Engineering, National Taiwan University, Taiwan, ROC
}

Accepted 11 December 2004

\begin{abstract}
Twenty young healthy adults walked and crossed obstacles of heights of $10 \%, 20 \%$ and $30 \%$ of their leg lengths and kinematics and kinetics of their gait analysed. Three-dimensional joint moments of the lower limb were calculated with inverse dynamics analysis. Peak moments and crossing moments (joint moments at the time when the swing foot is above the obstacle) were obtained. Most of the peak moments of the supporting limb joints did not show the same height effects as the corresponding crossing moment components. This suggests that the true effects of obstacle heights on the joint kinetics during obstacle crossing cannot be obtained solely through comparisons of peak moments. For inter-limb comparisons the peak and crossing moments were different, suggesting that the leading and trailing limb adopted different kinetic strategies during obstacle crossing. The findings may help to shed light on further investigation on the motor control strategies of the central nervous system for obstacle crossing.
\end{abstract}

(C) 2004 Elsevier B.V. All rights reserved.

Keywords: Gait; Obstacles; Joint moments; Lower limb

\section{Introduction}

Tripping over obstacles often leads to physical injuries, a common problem among the elderly [1-3]. A successful and safe obstacle crossing requires not only sufficient foot clearance of the swing limb but also the stability of the body provided mainly by the stance limb. Knowledge of the mechanics of the locomotor system and control strategies adopted during this activity is helpful for the understanding and identification of the risk factors of tripping which are important for prevention of falls [4-6]. There have been many studies investigating the kinematics of the lower extremities during obstacle crossing [7-16]. Kinematic indices such as clearance, horizontal distance and joint angles have been used to study the strategies when crossing obstacles of different heights. However, the kinetics of the stance limb that is associated with the stability of the body has not been studied as extensively.

\footnotetext{
* Corresponding author. Tel.: +886 2 33653335; fax: +886233653335. E-mail address: twlu@ntu.edu.tw (T.-W. Lu).
}

The majority of previous studies on joint kinetics during obstacle crossing have been limited to present sagittal components $[8,11,14]$. Although these studies contributed to the establishment of a general picture of strategies utilized during obstacle crossing in the sagittal plane, more insight into the mechanics and control strategies of this activity for future clinical applications requires a detailed three-dimensional (3D) kinetic analysis of the individual joints of both the leading and trailing lower limb. Chou and Draganich $[13,17]$ studied the changes of the 3D kinetics of the standing trailing limb when stepping over obstacles of four different heights (51, 102, 153, $204 \mathrm{~mm}$ ). McFadyen and Prince [16] described the 3D kinetics of the trailing stance limb during the avoidance of an obstacle of $117.5 \mathrm{~mm}$ in height. There seems to be no study in the literature reporting the 3D kinetics of the leading limb while the trailing limb is crossing the obstacle and comparing the roles of the leading and trailing limb in supporting the body during obstacle avoidance.

Key features of the joint moment curves are usually extracted for subsequent statistical analyses to study the effects of obstacle or crossing conditions on the lower limb kinetics. Previous studies $[13,16,17]$ have used peak values 
of the joint moments in the trailing limb for statistical analyses. Although changes of peak moments may reflect, to a certain extent, the mechanical adaptations to different test conditions, there are two limitations that should be considered. Firstly, peak values can occur at different points in time during obstacle crossing, not only for moments in different joints, but also for different moment components of an individual joint. Secondly, peak joint moments that occur before the swing foot is above the obstacle may have causal effects on the control of both the standing and swing limb but can be very different from joint moments at the time when the swing foot is above the obstacle, here referred to as crossing moments. The crossing moments seem to provide more direct information on how the movement of the body segments is modulated to avoid the obstacle. Therefore, apart from the peak moments, the crossing moments at the joints can be an important index for the study of the strategies of obstacle avoidance. However, no previous studies have examined crossing moments during obstacle crossing.

Studies on the kinetics of obstacle crossing have so far focused on that of the trailing limb [8,13,14,16,17]. However, it is noted that the toe clearances of the leading and trailing limb are similar [18]. Moreover, a person has no visual cue when their trailing limb is crossing the obstacle, increasing the chance of tripping over the obstacle. Therefore, data on the kinetics of the supporting leading limb as well as the kinematics of the crossing trailing limb are helpful for bridging the gap in the existing knowledge of control and coordination of the standing and swing limb.

The purposes of the present study were to investigate the control strategies adopted by both the leading and trailing limb when crossing obstacles in normal young adults by comparing the peak and crossing moments at each joint and to study the effects of obstacle heights on these strategies.

\section{Materials and methods}

Twenty young adults (12 males and 8 females; age, $23 \pm 3$ years; height, $172.6 \pm 6.7 \mathrm{~cm}$; weight, $66.4 \pm$ $10.6 \mathrm{~kg}$ ) participated in the present study having given informed consent. The study was approved by the Institutional Human Research Ethics Committee. Subjects were free of neuromusculoskeletal dysfunction and had normal corrected vision. Twenty-eight infrared retro reflective markers were used for the pelvis and lower extremities (ASIS's, PSIS's) and each thigh (greater trochanter, mid-thigh, medial and later epicondyles), shank (head of fibula, tibial tuberosity, medial and lateral malleolus) and foot (navicular tuberosity, fifth metatarsal base, big toe and heel). Another 10 markers were used on the acromion processes, medial and lateral humeral epicondyles, and the ulnar styloids. Each subject walked at a selfselected pace and crossed a height-adjustable obstacle made of an aluminium tube placed across a metal frame. Two additional markers were placed on each end of the tube to define the position of the obstacle. The subjects were allowed to familiarize themselves with the walkway before experimental data were taken. Kinematic data were measured with a seven camera motion analysis system (Vicon 512, Oxford Metrics, U.K.). Two force plates (AMTI, Advanced Mechanical Technology, U.S.A.) were placed on either side of the obstacle so that the ground reaction forces (GRF) of each foot could be measured. The crossing stride (CS) of the crossing limb was defined by using the measured force plate and kinematic data. The crossing stride of the leading limb began at toe-off before the obstacle and ended at toe-off after clearing the obstacle, while that for the trailing limb was defined as heelstrike before the obstacle to heelstrike after clearing the obstacle. Test conditions included unobstructed gait and crossing obstacles of three different heights $(10 \%, 20 \%$ and $30 \%$ of leg length) for both limbs. For all conditions, subjects were instructed to walk along the walkway and step over the obstacle when necessary. Six trials, three for each side, for each condition were recorded for each subject.

For dynamic analysis the pelvis-leg apparatus was modelled as a 7-link system, each link (limb segment) embedded with an orthogonal coordinate system with the positive $x$-axis directed anteriorly, the positive $y$-axis superiorly and the positive $z$-axis to the right. A cardanic rotation sequence $(Z-X-Y)$ was used to describe the rotational movements of each joint [19]. With the measured GRF and kinematic data, intersegmental internal forces and moments at the joints of the lower limbs during obstacle crossing were calculated using inverse dynamics. Inertial properties for each body segment - mass, centre of mass and moment of inertia were obtained using Dempster's coefficients [20]. Vertical positions of the centre of mass (COM) of the whole body were calculated as the weighted sum of those of the 12 body segments (trunk-head-neck, upper arms, forearm-hands, pelvis, thighs, shanks and feet). All the calculated moments were normalized to body weight and leg length (distance between ipsilateral ASIS and medial malleolus). For each trial, peak and crossing moments of each of the joints in the leading and trailing limb during stance phase were extracted from the calculated joint moment curves. The peak and crossing moments of the six trials (three for each side) for each condition of each subject were then averaged for subsequent statistical analysis. The calculated variables for different obstacle heights were analyzed using repeated measures analysis of variance (RMANOVA) with polynomial test to determine the trend (linear and quadratic) [21]. A significant level was set at $\alpha=0.05$. For comparisons between the leading and trailing limb a paired $t$-test $(\alpha=0.05)$ was used. SPSS version 10.0 (SPSS Inc., Chicago, IL) was used for all statistical analyses.

\section{Results}

The ensemble-averaged joint moments for the leading and trailing limb when crossing obstacles of $20 \%$ leg lengths 


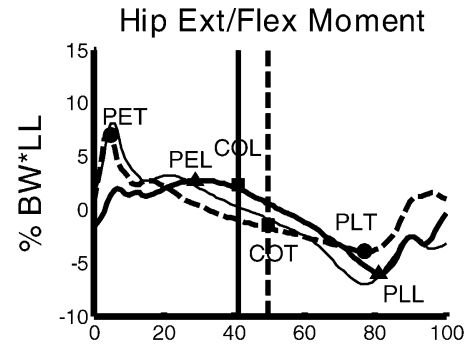

(a) Knee Ext/Flex Moment

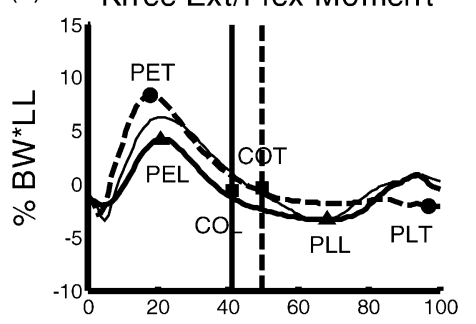

(b) Ankle Plant/Dorsi Moment

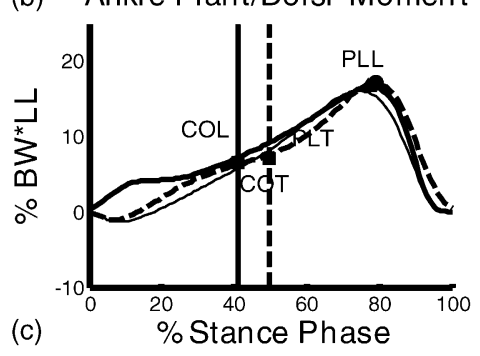

Hip Abd/Add Moment

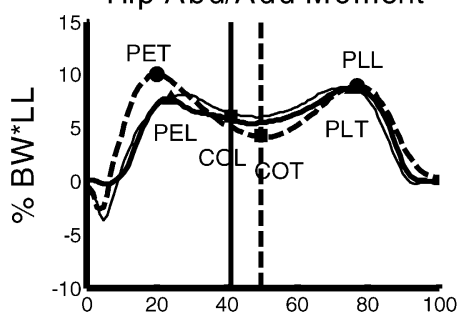

Knee $\mathrm{Abd} /$ Add Moment

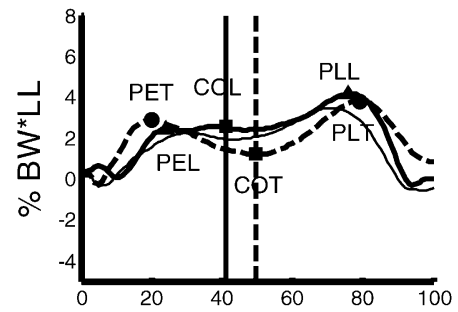

Ankle Abd/Add Moment

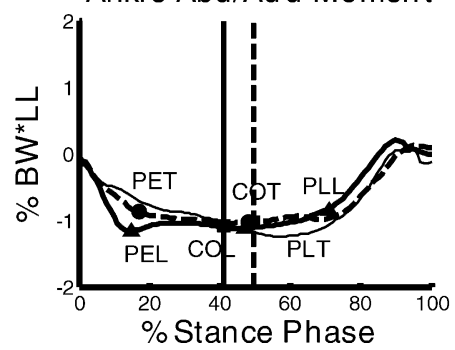

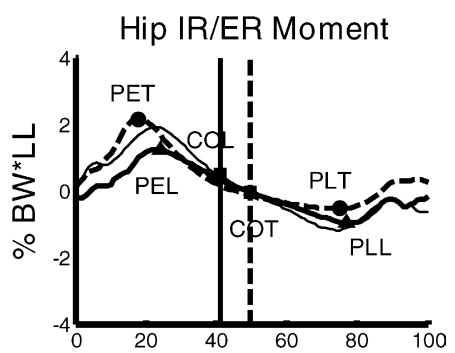

Knee IR/ER Moment

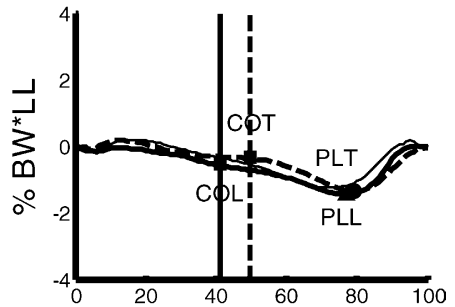

Ankle IR/ER Moment

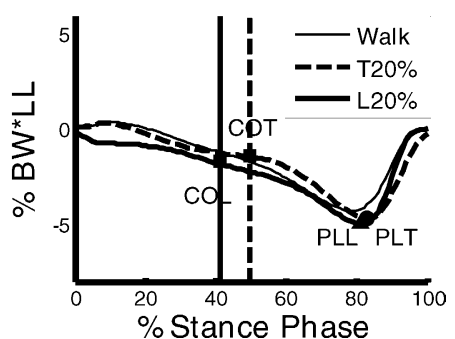

Fig. 1. Ensemble-averaged three-dimensional joint moments at the (a) hip, (b) knee and (c) ankle joints of the leading (solid) and trailing (dashed) limb during unobstructed gait (thin curves) and when crossing obstacles of $20 \%$ leg length (thick curves) (PEL and PET: peak moments in the early stance of the leading and trailing limb, respectively; PLL and PLT: peak moments in the late stance of the leading and trailing limb, respectively; COL and COT: crossing moments of the leading and trailing limb, respectively).

and during unobstructed gait are shown in Fig. 1. Similar patterns were also found for the $10 \%$ and $30 \%$ conditions. Peak moments for the leading limb were denoted as PEL during early stance and PLL during late stance while those for the trailing limb were PET and PLT, respectively. In all cases, the PELs and PETs occurred before the swing limb was above the obstacle and the PLLs and PLTs after. For the internal/external rotator moment curves of the knee and ankle joint and the plantar/dorsi flexor moment curve of the ankle joint, only peaks during late stance were selected. The crossing moments of the leading limb (COL) occurred at $39.1 \pm 3.3 \%, 41.1 \pm 3.1 \%$ and $43.6 \pm 2.8 \%$ stance phase for $10 \%, 20 \%$ and $30 \%$ conditions, respectively. Corresponding times for the crossing moments of the trailing limb (COT) were $52 \pm 2.7 \%, 49.5 \pm 2.9 \%$ and $47.1 \pm 2.6 \%$ stance phase. The time of the COL exhibited a linearly increasing trend with increasing height $(p<0.05)$ while that of the COT a linearly decreasing trend $(p<0.05)$.

Peak moments at different joints occurred at different points in time during obstacle crossing. The time differences of the occurrence of the peak moments at the hip and knee ranged from $1.1 \pm 0.9 \% \mathrm{CS}$ in the frontal component to $13.4 \pm 5.9 \% \mathrm{CS}$ in the sagittal component. The differences between the hip and ankle ranged from $1.9 \pm 1.8 \% \mathrm{CS}$ in the sagittal component to $10.3 \pm 2.5 \% \mathrm{CS}$ in the transverse component. Different components of the peak moments at a joint also occurred at different instances in time. At the hip joint the time differences of occurrence between any two components ranged from $1.6 \pm 2.0 \% \mathrm{CS}$ between frontal and transverse components to $10.3 \pm 1.7 \%$ CS between frontal and sagittal components. The differences for the knee ranged from $1.4 \pm 1.4 \% \mathrm{CS}$ between frontal and transverse components to $9.1 \pm 5.7 \%$ CS between transverse and sagittal components, while those for the ankle ranged from $1.5 \pm 1.1 \%$ CS between transverse and sagittal components to $13.3 \pm 6.8 \% \mathrm{CS}$ between frontal and transverse components.

Comparisons of the peak moments at the hip between leading and trailing limb for different obstacle heights are shown in Fig. 2. Corresponding data for the knee and ankle joints are given in Figs. 3 and 4, respectively. Significant differences between leading and trailing limb for the same height $(\alpha=0.05)$ are indicated in the figures. Comparisons of the crossing moments at the hip, knee and ankle joints between the leading and trailing limbs for different obstacle heights are shown in Figs. 5-7, respectively. Height effects on the peak and crossing moments at the joints in both leading and trailing limbs, in terms of results of trend analysis, are 


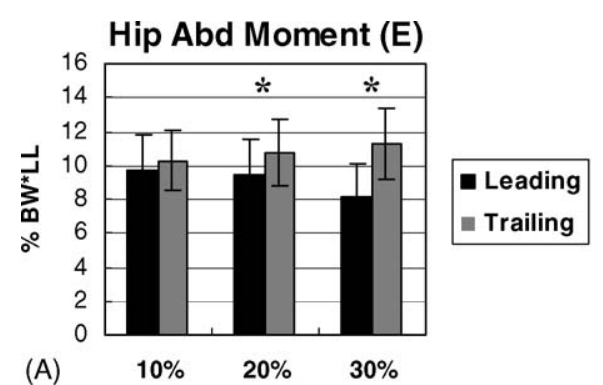

Hip Ext Moment (E)

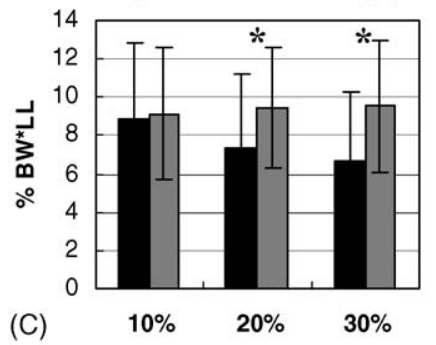

Hip IR Moment (E)

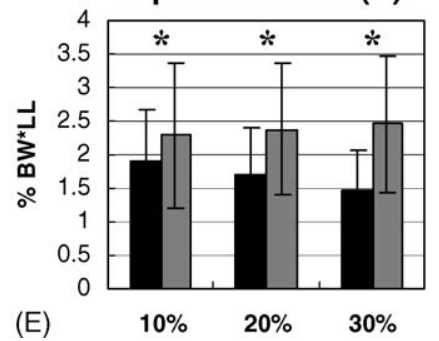

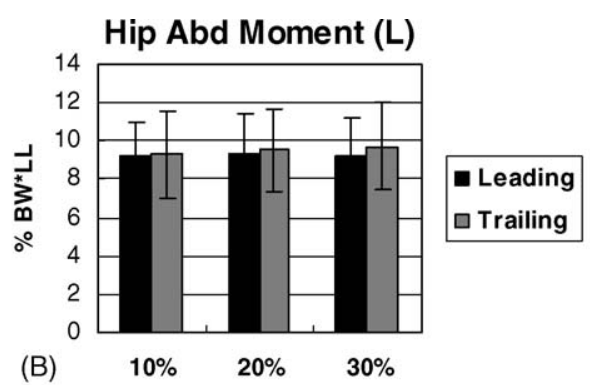

Hip Flex Moment (L)

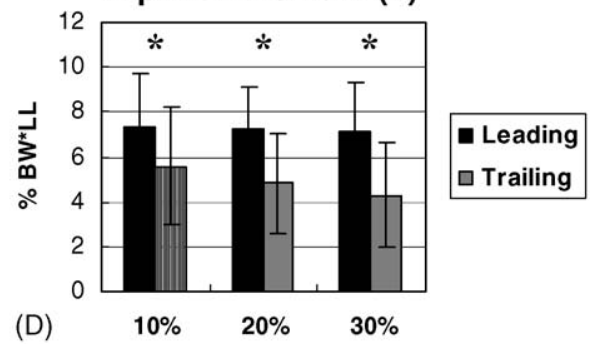

Hip ER Moment (L)

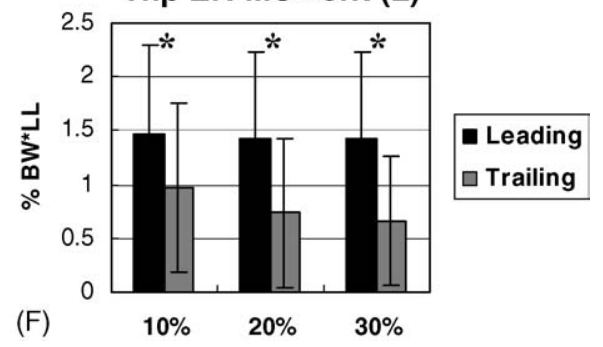

Fig. 2. Mean peak moments of the hip joint during early stance (E) and late stance (L) for the leading and trailing limb $(N=20)$. Standard deviations are shown as error bars. An asterisk indicates significant differences between the leading and trailing limb $(p<0.05)$.

shown in Table 1. The height effects for the crossing moments for both limbs were quite different from those for the peak moments during both early and late stance.

Table 1

Summary of the significant height effects on the peak and crossing moments at the joints in the leading and trailing limb

\begin{tabular}{|c|c|c|c|c|c|c|}
\hline \multirow[t]{2}{*}{ Joint moments } & \multicolumn{3}{|c|}{ Leading } & \multicolumn{3}{|c|}{ Trailing } \\
\hline & PEL & $\mathrm{COL}$ & PLL & PET & COT & PLT \\
\hline \multicolumn{7}{|l|}{ Hip } \\
\hline $\mathrm{B} / \mathrm{D}$ & $\mathrm{B} \downarrow$ & $\mathrm{B} \downarrow$ & - & $\mathrm{B} \uparrow$ & $\mathrm{B} \downarrow$ & - \\
\hline $\mathrm{T} / \mathrm{F}$ & $\mathrm{T} \downarrow$ & - & - & - & - & $\mathrm{F} \downarrow$ \\
\hline $\mathrm{I} / \mathrm{E}$ & $\mathrm{I} \downarrow$ & $\mathrm{I} \downarrow$ & - & - & $\mathrm{I} \downarrow$ & $\mathrm{E} \downarrow$ \\
\hline \multicolumn{7}{|l|}{ Knee } \\
\hline $\mathrm{B} / \mathrm{D}$ & - & - & $\mathrm{B} \uparrow$ & - & $\mathrm{B} \downarrow$ & $\mathrm{B} \uparrow$ \\
\hline $\mathrm{T} / \mathrm{F}$ & $\mathrm{T} \downarrow$ & $\mathrm{F} \uparrow$ & $\mathrm{F} \uparrow$ & $\mathrm{T} \uparrow$ & $\mathrm{F} \downarrow$ & - \\
\hline $\mathrm{I} / \mathrm{E}$ & - & - & $\mathrm{E} \uparrow$ & - & $\mathrm{E} \downarrow$ & - \\
\hline \multicolumn{7}{|l|}{ Ankle } \\
\hline $\mathrm{B} / \mathrm{D}$ & - & - & - & - & - & - \\
\hline $\mathrm{T} / \mathrm{F}$ & - & - & $\mathrm{T} \uparrow$ & - & $\mathrm{T} \downarrow$ & $\mathrm{T} \uparrow$ \\
\hline $\mathrm{I} / \mathrm{E}$ & - & $\mathrm{E} \uparrow$ & $\mathrm{E} \uparrow$ & - & $\mathrm{E} \downarrow$ & $\mathrm{E} \uparrow$ \\
\hline
\end{tabular}

$\uparrow$, Joint moments increase linearly with increasing obstacle height; $\downarrow$, joint moments decrease linearly with increasing obstacle height; -, no height effect; B, abductor; D, adductor; T, extensor/planar flexor; F, flexor/dorsi flexor; I, internal rotator; E, external rotator.
The mean vertical trajectories of the COM during the trailing crossing stride for all conditions are shown in Fig. 8. It was found that the height of the COM increased significantly $(p<0.05)$ with increasing obstacle height when the leading limb was crossing. The same results were also found when the trailing limb was crossing. The mean vertical GRFs of both limbs during stance phase are shown in Fig. 9. It was found that vertical GRFs corresponding to the COT, here referred to as CGRFT, decreased linearly with increasing height $(p<0.05)$. However, those corresponding to the COL, here referred to as CGRFL, did not show any height effect $(p>0.05)$.

\section{Discussion}

\subsection{Height effect on the trailing peak moments}

The patterns of the joint moment curves for the trailing limb calculated in the present study are similar to those reported by others $[13,14,16,17]$. The only study that investigated the effects of obstacle heights on the joint kinetics of the trailing limb was by Chou and Draganich [13], focusing on peak moments of the trailing limb when 
Knee Abd Moment (E)

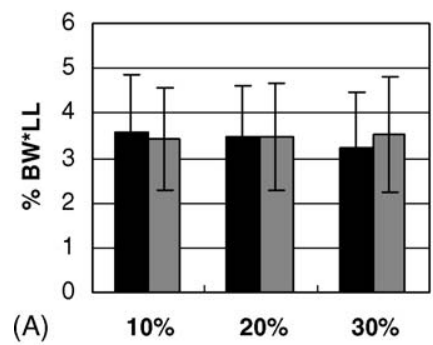

Knee Ext Moment (E)

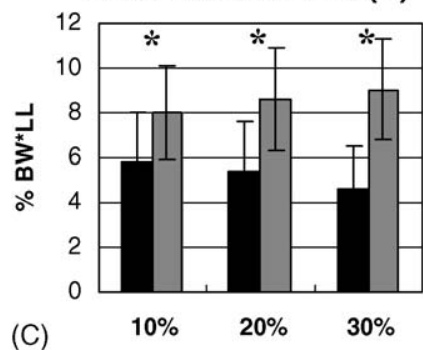

Knee Abd Moment (L)

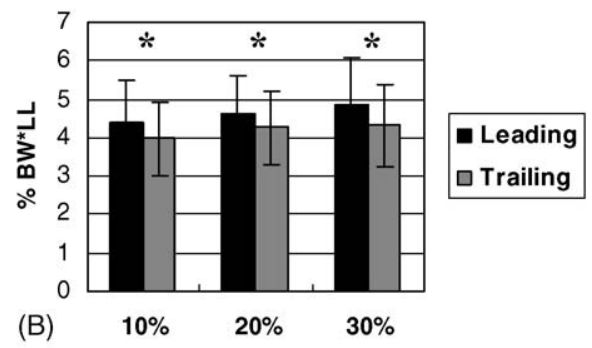

\section{Knee Flex Moment (L)}

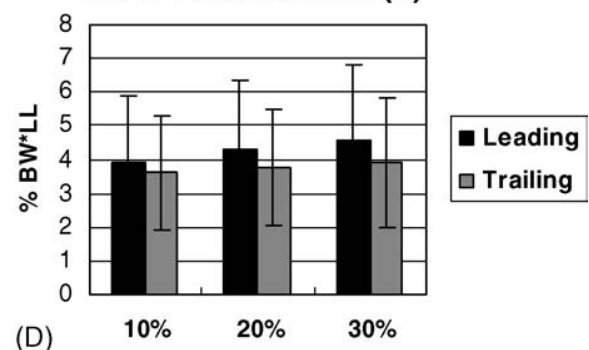

\section{Knee ER Moment (L)}

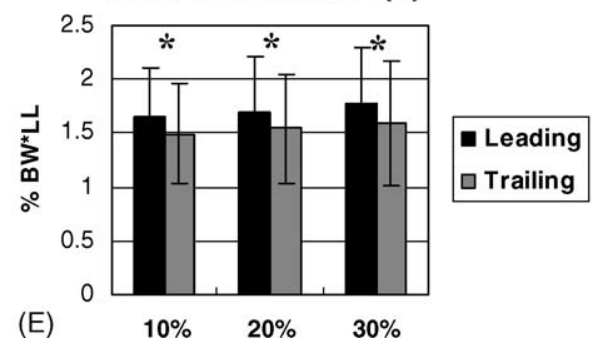

Fig. 3. Mean peak moments of the knee joint during early stance (E) and late stance (L) for the leading and trailing limb $(N=20)$. Standard deviations are shown as error bars. An asterisk indicates significant differences between the leading and trailing limb $(p<0.05)$.

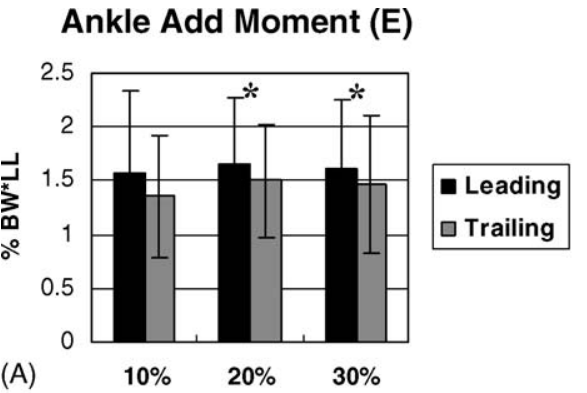

Ankle Plant Moment (L)

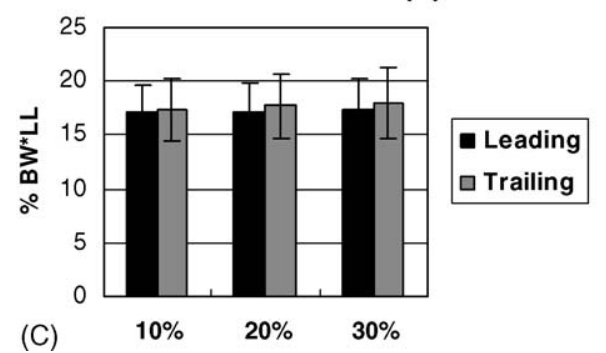

Ankle Add Moment (L)

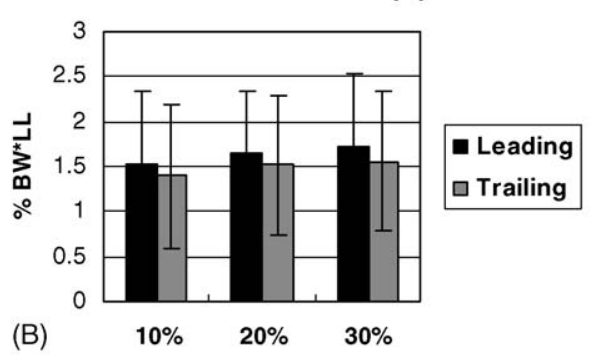

Ankle ER Moment (L)

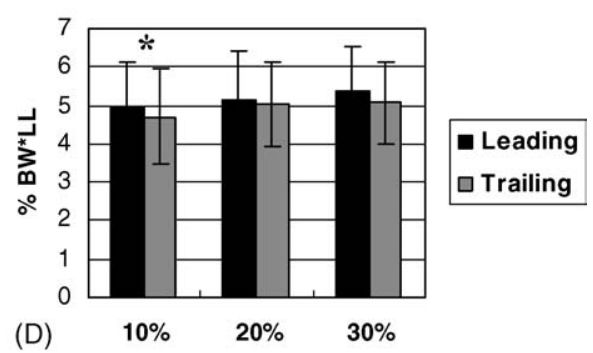

Fig. 4. Mean peak moments of the ankle joint during early stance (E) and late stance (L) for the leading and trailing limb $(N=20)$. Standard deviations are shown as error bars. An asterisk indicates significant differences between the leading and trailing limb $(p<0.05)$. 


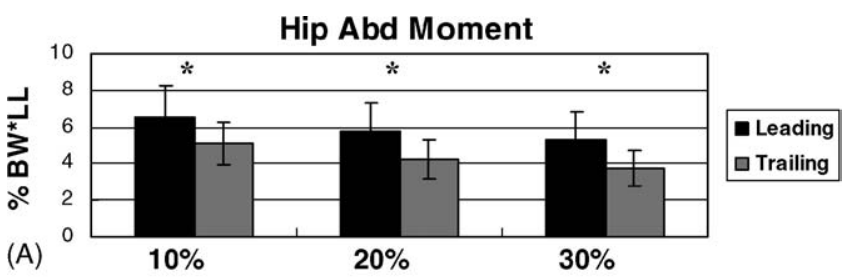

Hip Ext Moment

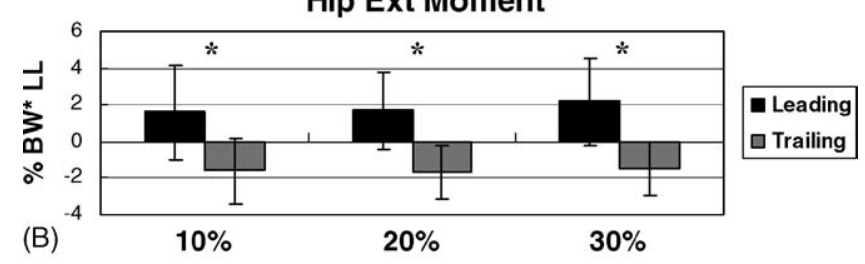

Hip IR Moment

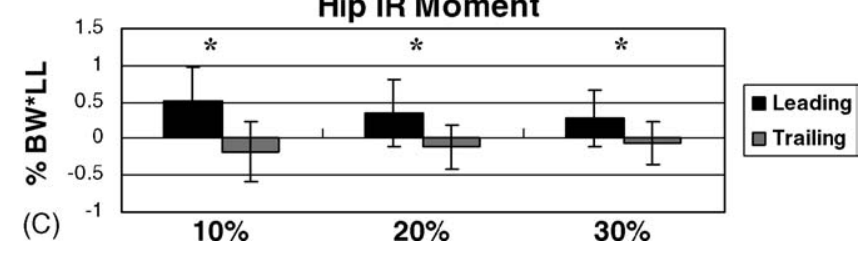

Fig. 5. Mean crossing moments of the hip joint when the swing toe is above the obstacle for the leading and trailing $\operatorname{limb}(N=20)$. Standard deviations are shown as error bars. An asterisk indicates significant differences between the leading and trailing limb $(p<0.05)$.

crossing obstacles of four fixed heights $(51,102,153$ and $204 \mathrm{~mm}$ ). In the present study, the trailing hip flexor PLT was found to decrease with obstacle height while knee extensor PET, abductor PLT and ankle plantar flexor PLT

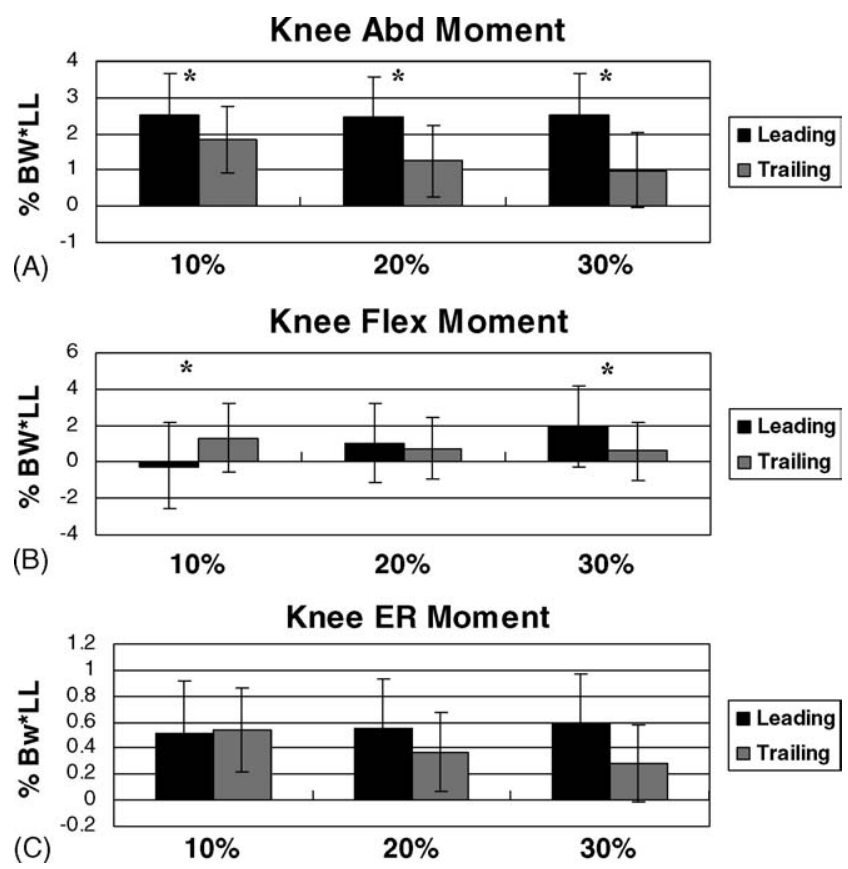

Fig. 6. Mean crossing moments of the knee joint when the swing toe is above the obstacle for the leading and trailing limb $(N=20)$. Standard deviations are shown as error bars. An asterisk indicates significant differences between the leading and trailing limb $(p<0.05)$.
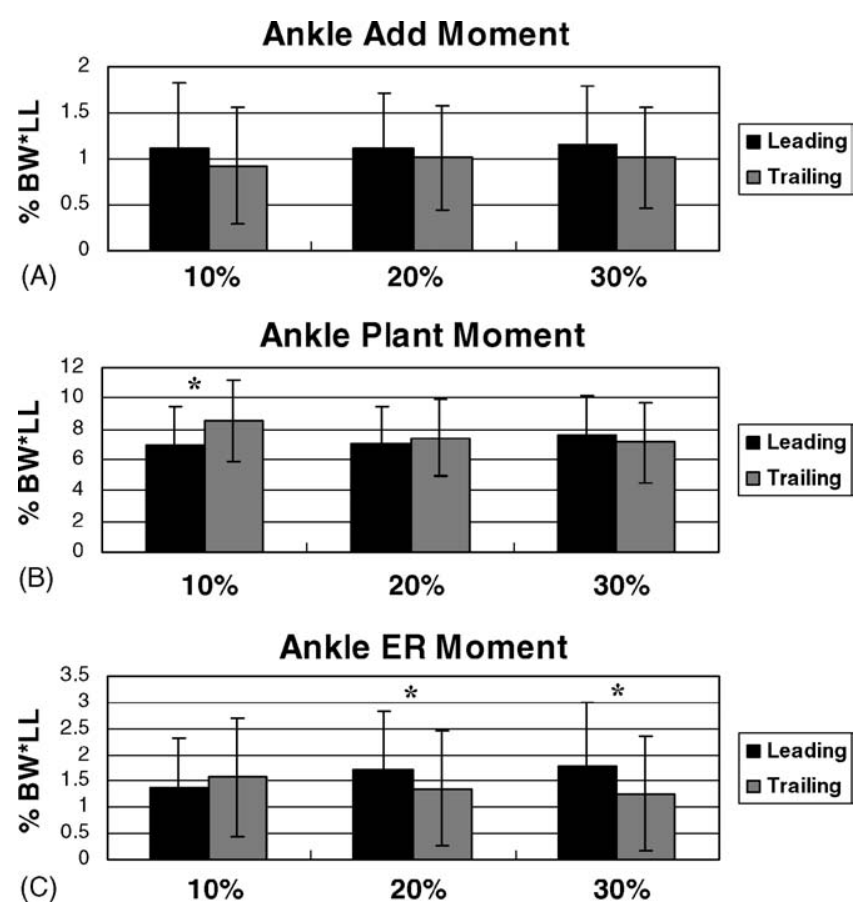

Fig. 7. Mean crossing moments of the ankle joint when the swing toe is above the obstacle for the leading and trailing limb $(N=20)$. Standard deviations are shown as error bars. An asterisk indicates significant differences between the leading and trailing $\operatorname{limb}(p<0.05)$.

increased with increased height, in agreement with Chou and Draganich [13], Table 1. Apart from these moment components, height effects were also found in hip abductor PET, external rotator PLT and ankle external rotator PLT in the present study, Table 1 . This could possibly be related to the main methodological difference between the two studies that obstacles normalized to leg lengths were selected in the present study while fixed heights were used by Chou and

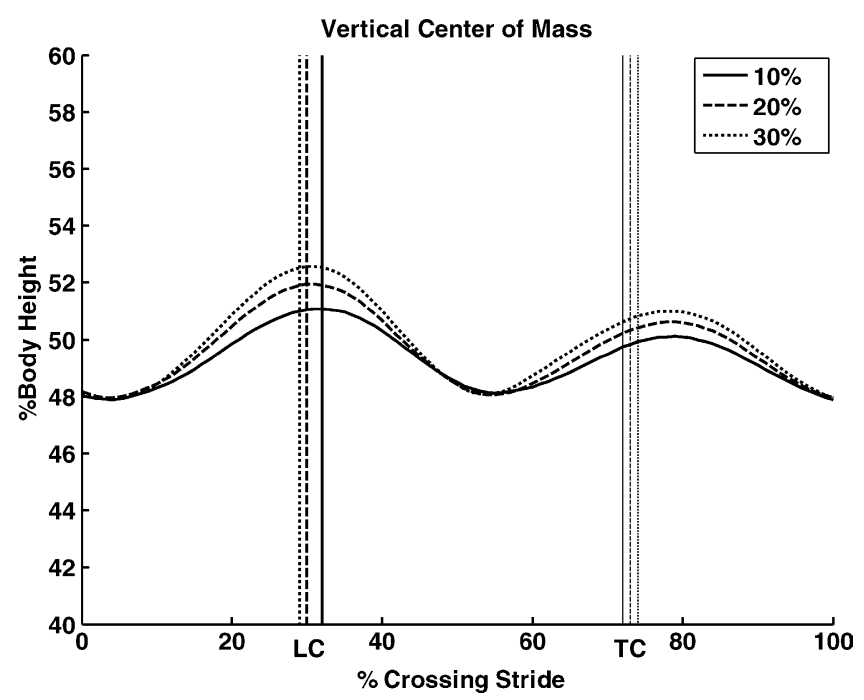

Fig. 8. Mean vertical displacements of the centre of body mass during obstacle crossing for $10 \%$ (solid), $20 \%$ (dashed) and $30 \%$ (dotted) conditions $(N=20)$. The instances when the leading foot is above the three obstacles (LC) are indicated with thick vertical lines while those for the trailing foot with thin lines (TC). 


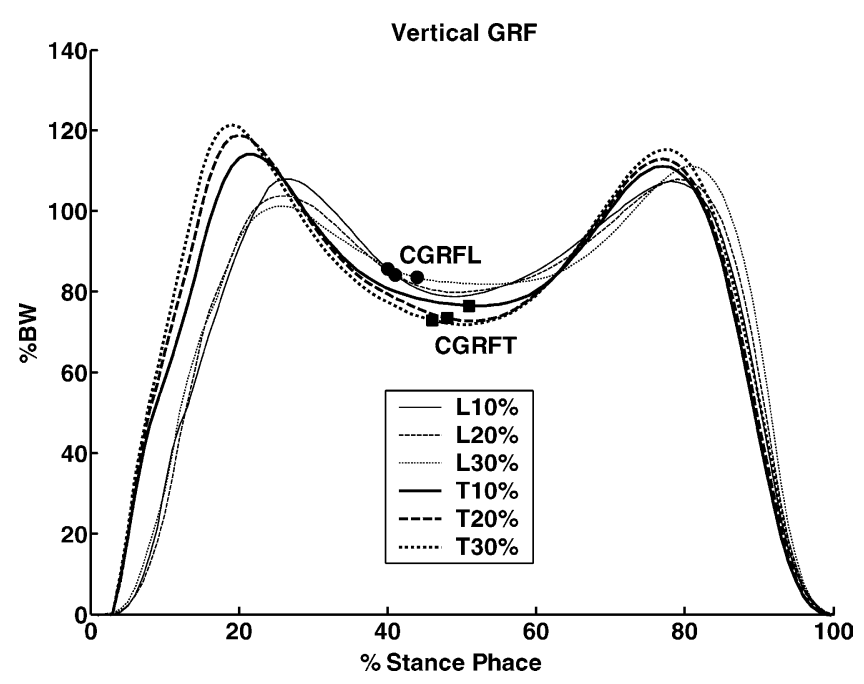

Fig. 9. Mean vertical components of the ground reaction forces of the leading (thin curve) and trailing (thick curve) limb during the stance phase when crossing obstacles of $10 \%$ (solid lines), $20 \%$ (dashed lines) and $30 \%$ (dotted lines) of leg lengths. Also shown are CGRFL in solid circles and CGRFT in solid squares.

Draganich [13]. Sparrow et al. [12] suggested that obstacles should be adjusted for subjects' leg lengths because of intersubject size differences. It is possible that hip abductor PET, external rotator PLT and ankle external rotator PLT are more sensitive to subject size differences.

\subsection{Height effect on the leading peak moments}

The leading limb provides the necessary stability for the trailing swing limb to cross the obstacle with sufficient clearance. The study of the kinematics of the trailing swing limb requires a complete knowledge of the kinetics of the leading stance limb. However, no study has investigated the kinetics of the leading limb and possible obstacle height effects. In the present study, during early stance, all the PEL components at the hip and the knee extensor PEL were found to decrease linearly with increasing height while other components remained unaffected. During late stance, all the PLL components at the knee and ankle, except the ankle adductor PLL, were found to increase linearly with increasing height while other components remained unaffected. Since joint moments were provided mainly by muscles, these results suggested that the hip muscles played a major role in modulating the joint loadings in response to the change in obstacle height during early stance while the knee and ankle muscles did so during late stance.

\subsection{Height effect on the trailing crossing moments}

Although peak moments of the supporting limb may reflect the overall mechanical demands on joints during the stance phase of obstacle crossing, knowledge of the roles played by the trailing and leading limb in supporting the body when the swing foot is over the obstacle requires information of the crossing moments. Moreover, peak moments were found to occur at different points in time during obstacle crossing, not only for moments at different joints, but also for different moment components of an individual joint. Since obstacle crossing is predominately a multi-joint movement, the use of the peak moments for the interpretation of the control of the lower limb and interjoint coordination should consider the time of occurrence of individual moment components. Unfortunately, this increases the difficulty in results interpretation. An alternative may be the use of crossing moments which always occur at the time when the swing foot is above the obstacle, allowing direct inter-joint comparisons.

For the trailing limb, all components of the crossing moments at the three joints showed linearly decreasing trends $(p<0.05)$ with increasing obstacle height except for those of the hip flexors and ankle adductors which remained unaffected, Table 1. Analysis of the displacements of the centre of body mass (COM) showed that the height of the $\mathrm{COM}$ reached a maximum when the swing foot was above the obstacle and it increased significantly $(p<0.05)$ with increasing obstacle height, Fig. 8. This suggests that straighter and closer-to-vertical limb positions were needed for higher obstacles. On the other hand, the vertical GRFs were found to decrease linearly $(p<0.05)$ with increasing obstacle height, Fig. 9. It seems that both factors contributed to the noted decreasing crossing moments with increasing obstacle heights.

\subsection{Height effect on the leading crossing moments}

For the leading limb, with increasing obstacle height, the crossing moments of the hip abductors and internal rotators decreased linearly $(p<0.05)$ while linear increasing trend was found for the knee flexors and ankle external rotators $(p<0.05)$. Hip abductor and internal rotator crossing moments tended to accelerate the COM of the body upward while the body was moving forward. The crossing moments of the knee flexors and external ankle rotators produced the reverse effects. The decrease of the former and increase of the latter both contributed to the deceleration of the COM and the control of the forward and upward momentum of the body gained at an earlier stage of obstacle crossing. This may help to prevent instability of the whole body when the swing foot is above the obstacle.

\subsection{Peak moments versus crossing moments}

It was found that peak moments in both limbs did not show the same height effects as the corresponding crossing moments did for all moment components of all joints, Table 1. This suggests that the true effects of obstacle heights on the joint kinetics during obstacle crossing cannot be obtained solely through comparisons of peak moments. It is noted that joint peak moments occurred near the trough of 
the COM vertical trajectories, or during the course of the rise of the COM, indicating that the work needed for the forward and upward movement of the COM was done by these peak moments at appropriate time instances. Therefore, the peak moments were needed mainly for propulsion of the body forward and upward. On the other hand, crossing moments occurred either at the peak of the COM trajectories for the trailing limb or near the peak for the leading limb, Fig. 8. Since higher the COM, the less the stability of the body during movement, it may be postulated that the joint crossing moments were mainly used for precision control of the limbs to ensure a successful avoidance of the obstacle. These smaller crossing moments may also suggest that the muscles have more room to act in response to any unexpected disturbances during the crossing.

\subsection{Inter-limb comparison of the peak moments}

Comparisons of the joint kinetics between the leading and trailing limb may be helpful for the understanding of the muscular demand during different phases of obstacle crossing. Most of the peak moment components in the trailing joints during early stance were found to be bigger than the corresponding values in the leading joints (Figs. 24), suggesting that before the swing foot was above the obstacle the maximum muscular demands in the trailing limb were bigger than those of the leading limb. During late stance, however, most of the peak trailing limb moment components were smaller than those of the leading limb. These results suggest that different muscular efforts were required for the leading and trailing limb to meet different supporting and propulsion requirements during the stance phases in obstacle crossing.

\subsection{Inter-limb comparison of the crossing moments}

The height effects of the crossing moments in the leading limb were found to be different from those of the trailing limb. The leading limb required hip extensor and internal rotator moments for all heights and a knee extensor moment in the $10 \%$ condition. In contrast, hip flexor and external rotator moments were needed in the trailing limb. Most of the crossing moment components in the leading limb were statistically greater than those in the trailing limb for all heights (Figs. 5-7). These results may be explained by the differences in the CGRFL and CGRFT (Fig. 9). Compared to CGRFT, CGRFL was significantly bigger for all heights (Fig. 9). In the present study, the CGRFT for all heights occurred around the trough of the trailing GRF curve while CGRFL occurred earlier and higher than the trough of the leading GRF curve. Begg et al. [22] found that the values of the troughs of the vertical component curves of the leading limb ground reaction forces were significantly bigger than those for the trailing limb when crossing obstacles of $20 \%$ and 30\% leg length in height. The same trend was also found in the $10 \%$ obstacle. Based on these data, Begg et al. [22] suggested that different kinetic strategies were adopted by the leading and trailing limb during obstacle crossing. Their results support the present findings that CGRFL was bigger than CGRFT, which together with results on crossing moments provides more direct support of the hypothesis that the leading and trailing limb adopts different kinetic strategies during obstacle crossing.

\section{Conclusions}

A successful and safe obstacle crossing requires not only sufficient foot clearance of the swing limb but also the stability of the body provided mainly by the stance limb. Therefore, data on the joint moments of the supporting limb are necessary for the study of its roles in maintaining body stability during obstacle crossing. It was found that most of the peak moments of the supporting limb joints did not show the same height effects as the corresponding crossing moment components. This suggests that the true effects of obstacle heights on the joint kinetics during obstacle crossing cannot be obtained solely through comparisons of peak moments. The peak moments are needed mainly for propulsion of the body forward and upward while the joint crossing moments are mainly used for precision control of the limbs to ensure a successful avoidance of the obstacle. For inter-limb comparisons, both the peak and crossing moments were different, suggesting that the leading and trailing limb adopted different kinetic strategies during obstacle crossing. Results of the present study on normal young adults helped bridging some of the gaps in the current knowledge of lower limb mechanics during obstacle crossing and can be used as a baseline in the evaluation of patients or the elderly. They are helpful for the understanding and identification of the risk factors of tripping which are important for developing effective interventions for avoiding falls. The findings may also help to shed light on further investigation on the motor control strategies of the central nervous system for obstacle crossing.

\section{References}

[1] Tinetti ME, Speechley M, Ginter SF. Risk factors for falls among elderly persons living in the community. $\mathrm{N}$ Engl $\mathrm{J}$ Med 1988;319:1701-7.

[2] Tinetti ME, Speechley M. Prevention of falls among the elderly. N Engl J Med 1989;320:1055-9.

[3] Sattin RW. Falls among older persons: a public health perspective. Ann Rev Public Health 1992;13:489-508.

[4] Chou LS, Draganich LF. Placing the trailing foot closer to an obstacle reduces flexion of the hip, knee and ankle to increase the risk of tripping. J Biomech 1998;31:685-91.

[5] Draganich LF, Kuo CE. The effects of walking speed on obstacle crossing in healthy young and healthy older adults. J Biomech 2004;37:889-96.

[6] Schrodt LA, Mercer VS, Guiuliani CA, Hartman M. Characteristics of stepping over an obstacle in community dwelling older adults under dual-task conditions. Gait Posture 2004;19:279-87. 
[7] Chen HS, Ashton-Miller JA, Alexander NB, Schultz AB. Stepping over obstacles: gait patterns of healthy young and old adults. J Gerontol 1991;46:196-203.

[8] McFadyen BJ. Anticipatory locomotor adjustment during obstructed human walking. Neurosci Res Commun 1991;9:37-44.

[9] Patla AE, Prentice SD, Robinson C, Neufeld J. Visual control of locomotion: strategies for changing direction and for going over obstacles. J Exp Psychol Hum Percept Perform 1991;17: 603-34.

[10] Patla AE, Rietdyk S. Visual control of limb trajectory over obstacles during locomotion: effect of obstacle height and width. Gait Posture 1993;1:45-60.

[11] Patla AE, Prentice SD. The role of active forces and intersegmental dynamics in the control of limb trajectory over obstacles during locomotion in humans. Exp Brain Res 1995;106:499-504.

[12] Sparrow W, Shinkfield AJ, Chow S, Begg R. Characteristics of gait in stepping over obstacles. Hum Mov Sci 1996;15:605-22.

[13] Chou LS, Draganich LF. Stepping over an obstacle increases the motions and moments of the joints of the trailing limb in young adults. J Biomech 1997;30:331-7.

[14] McFadyen BJ, Carnahan H. Anticipatory locomotor adjustments for accommodating versus avoiding level changes in humans. Exp Brain Res 1997;114:500-6.
[15] Austin GP, Garrett GE, Bohannon RW. Kinematic analysis of obstacle clearance during locomotion. Gait Posture 1999;10:109-20.

[16] McFadyen BJ, Prince F. Avoidance and accommodation of surface height changes by healthy, community-dwelling, young and elderly men. J Gerontol 2002;57:166-74.

[17] Chou LS, Draganich LF. Increasing obstacle height and decreasing toe-obstacle distance affect the joint moments of the stance limb differently when stepping over an obstacle. Gait Posture 1998;8:186204.

[18] Chen HL, Lu TW, Lin HC. Three-dimensional kinematic analysis of stepping over obstacles in young subjects. Biomed Eng Appl Basis Commun 2004;16:157-64.

[19] Cole GK, Nigg BM, Ronsky JL, Yeadon MR. Application of the joint coordinate system to three-dimensional joint attitude and movement representation: a standardization proposal. J Biomech Eng 1993;115: 344-9.

[20] Winter DA. Biomechanics and Motor Control of Human Movement, New York: John Wiley \& Sons, Inc., 1990.

[21] Liu Y. Analyzing RM ANOVA related data using SPSS10. Meas Phys Edu Exercise Sci 2002;6:43-60.

[22] Begg RK, Sparrow W, Lythgo N. Time-domain analysis of footground reaction forces in negotiating obstacles. Gait Posture 1998;7:99-109. 